\title{
Exploring the Impact of Modifiable Factors on Serum BDNF in Psychiatric Patients and Community Controls
}

This article was published in the following Dove Press journal:

Neuropsychiatric Disease and Treatment

\author{
Galen Chan (D) \\ Tea Rosic (D) ${ }^{2,3}$ \\ Stanislav Pasyk ${ }^{2}$ \\ Mahshid Dehghan ${ }^{4}$ \\ Zainab Samaan $\mathbb{D}^{2,3}$ \\ 'Michael G. DeGroote School of \\ Medicine, McMaster University, \\ Hamilton, Ontario, Canada; ${ }^{2}$ Department \\ of Psychiatry and Behavioral \\ Neurosciences, McMaster University, \\ Hamilton, Ontario, Canada; ${ }^{3}$ Department \\ of Health Research Methods, Evidence \\ and Impact, McMaster University, \\ Hamilton, ON, Canada; ${ }^{4}$ Population \\ Health Research Institute, McMaster \\ University, Hamilton, Ontario, Canada
}

Correspondence: Zainab Samaan Department of Psychiatry and Behavioural Neurosciences,

McMaster University, Hamilton, ON, Canada

Tel +| 905522 |I 55 ext. 39215

$\mathrm{Fax}+19055282814$

Email samaanz@mcmaster.ca
Background: Brain-derived neurotrophic factor (BDNF) has been a focus of psychiatric research for the past two decades. BDNF has been shown to impact neural function and development. Studies have investigated serum BDNF as a biomarker for psychiatric disorders such as depression and schizophrenia. In some studies, investigators attempt to control for variables such as smoking status, exercise, or diet. However, the relationship between these factors and BDNF is not clearly established. Furthermore, some studies have questioned whether a difference in the impact of BDNF exists between psychiatric and healthy populations.

Purpose: We aim to examine the association between serum BDNF levels and modifiable risk factors such as body mass index (BMI), smoking, exercise levels, and diet. Subsequently, we aim to examine whether the relationship between these risk factors and serum BDNF is different between psychiatric and control populations.

Patients and Methods: We use cross-sectional data from an age- and sex-matched casecontrol study of participants with psychiatric inpatients and community controls without psychiatric diagnoses. Participants completed comprehensive assessments at study enrolment including sociodemographic information, smoking status, exercise, diet, and BMI. Serum BDNF levels were collected from participants. Linear regression analysis was performed to determine the association between modifiable factors and serum BDNF level.

Results: A significant association was found between sedentary activity level and lower serum BDNF levels (Beta coefficient $=-2.49,95 \%$ confidence interval $[\mathrm{CI}]-4.70,-0.28, p=0.028$ ). Subgroup analysis demonstrated that this association held for psychiatric inpatients but not for community controls; it also held in females (Beta coefficient $=-3.18,95 \% \mathrm{CI}-6.29,-0.07, p=$ 0.045 ) but not in males (Beta coefficient $=-1.42,95 \% \mathrm{CI}-4.61,1.78, p=0.383$ ). Antidepressant use had a significantly different association between male (Beta coefficient $=3.20,95 \%$ CI 0.51 , $5.88, p=0.020$ ) and female subgroups (Beta coefficient $=-3.10,95 \% \mathrm{CI}-5.75,-0.46, p=$ 0.022). No significant association was found between other factors and serum BDNF.

Conclusion: Sedentary activity level may lead to lower serum BDNF levels in individuals with psychiatric diagnoses. Our findings support the notion that physical activity can provide a positive impact as part of treatment for psychiatric illness.

Keywords: BDNF, psychiatric disorders, physical activity, diet, behavioral risk factors, depression

\section{Introduction}

Brain-derived neurotrophic factor (BDNF) has been a focus of psychiatric research for most of the past two decades. A member of the neurotrophin family of proteins, 
BDNF has been shown to impact many facets of neural function and development, such as neurogenesis, neurodensity, long-term potentiation, and dendritic growth. ${ }^{1,2}$ The most abundant and broadly distributed neurotrophic factor within the adult brain, BDNF is found distributed throughout the central nervous system and circulating through the blood. ${ }^{3,4}$ BDNF is synthesized as a precursor protein (proBDNF) which is then cleaved and converted to mature BDNF and BDNF pro-peptide. Both proBDNF and mature BDNF have been noted to be important in several physiological functions and elicit opposing effects to each other via their receptors. Recent studies have hypothesized that alterations to the biosynthesis mechanisms modulating proBDNF and mature BDNF underlie the development of neurodegenerative conditions. ${ }^{5,6}$

With its established impact on neural function, previous studies have subsequently examined whether BDNF plays a role in neuropathological changes that result in the development of psychiatric conditions or concerns. Reduced BDNF levels have been associated with major depressive disorder, schizophrenia, dementia, and suicide. ${ }^{7-12}$ While BDNF is mainly active in the central nervous system, and while the exact mechanism relating serum and brain BDNF levels remains unclear, serum BDNF changes have been correlated with these conditions. ${ }^{13-15}$ Also of note, a particular BDNF gene polymorphism, the Val66Met polymorphism, has been associated with increased gene methylation and susceptibility to depression. ${ }^{16}$ However, many of these studies do not address confounding factors, such as smoking or obesity, and differing study protocols such as time of collection of biological samples. ${ }^{17}$ For example, in a systematic review by Eisen et al examining the associations between BDNF and suicidal behaviour, not all casecontrol studies measuring serum BDNF conducted adjusted analyses. ${ }^{17-19}$ Furthermore, some studies did not delineate between a recent suicide attempt or a lifetime history of suicide attempt, putting the time interval between the suicide attempt event and serum BDNF collection in question. ${ }^{17-20}$ In studies where these factors were adjusted to more closely examine the relationship between serum BDNF and schizophrenia, for example, no statistically significant difference was found, bringing previous results into question. $^{21}$

Several previous studies have investigated the association between risk factors such as age, ${ }^{22}$ exercise, ${ }^{23-29}$ dietary intake ${ }^{29}$ and obesity ${ }^{30,31}$ and an individual's BDNF levels. These factors have been postulated as modulators for various psychiatric disorders, and it was hypothesized that these factors may impact neuropsychiatric processes through BDNF pathways. ${ }^{16}$ However, limited sample sizes, lack of control groups for various variables, and lack of BDNF gene polymorphism data have all been limiting factors that contribute to the uncertainty of the results within the literature. Another concern within previous studies is a lack of standardized protocol when comparing results between studies; a meta-analysis by Molendijk et al noted a lack of control for confounding factors and limited sample sizes in a meta-analyses examining serum BDNF and its role as a biomarker for depression. $^{32}$

Questions have also been raised about whether these factors impact BDNF levels differently between psychiatric populations and healthy populations, which could suggest an underlying pathophysiology within the BDNF pathways within psychiatric patients. ${ }^{33}$ One meta-analysis on the effects of exercise on BDNF found a tentative $2: 1$ difference in mean effect sizes for BDNF level changes between these two populations. ${ }^{33}$ The meta-analysis further noted that more studies would need to be done to confirm the findings.

In a previous study from our group by Eisen et al (2016) which investigated various modifiable variables and their impact on serum BDNF levels in psychiatric patients (divided into those who attempted suicide and those who did not) and community controls, there was no significant association between risk factors such as age, sex, smoking status, or body mass index. ${ }^{34}$ However, the authors did not investigate whether the relationship between these variables and BDNF differed between participants with and without psychiatric disorders.

There remains uncertainty about the relationship between modifiable risk factors and serum BDNF levels and differences in individuals with and without psychiatric disorders. In the present study, we sought to investigate whether there is an association between serum BDNF levels and modifiable factors such as body mass index (BMI), smoking status, exercise levels, antidepressant medication use, and diet amongst individuals with and without psychiatric disorders using cross-sectional data from 311 participants.

\section{Methods}

\section{Study Design}

We used cross-sectional data collected in the Determinants of Suicidal Behavior: Conventional and Emergent Risk 
(DISCOVER) study, for which study methods have been previously published. ${ }^{35}$ Briefly, DISCOVER is an age- and sex-matched case-control study of participants with recent suicide attempts (within 1 month) admitted to hospital, psychiatric inpatients without a history of suicide attempts, and community controls without psychiatric diagnoses. Inclusion criteria were: males and females aged 18 years and older and able to provide informed consent and communicate in English. For the purposes of this study, a suicide attempt was defined as a self-directed injury with intent to die. Participants completed detailed assessments at study entry including demographic information, medical and psychiatric history, prescribed medications (including antidepressants), and received the MiniInternational Neuropsychiatric Interview ${ }^{36}$ administered by trained interviewers. We measured height and weight for every participant at the time of study enrolment in order to calculate body mass index (BMI). We also obtained self-report information on smoking status and exercise levels. Sedentary activity was defined as no reported physical activity at work or during leisure. We measured diet intake using a short Food Frequency Questionnaire (FFQ). We asked participants to report daily intake of main food groups, such as whole grains, refined grains, fruits vegetables and nuts. ${ }^{37}$ Overnight fasting blood samples, and after 30 minutes of clotting time were placed in tubes and spun for 15 minutes at 3000rpm until the blood was well separated. ${ }^{35}$ Samples were aliquoted, stored in cryovials within 2 hours of collection, and frozen in liquid nitrogen. ${ }^{35}$ Serum BDNF level was assayed using the Quantikine ${ }^{\circledR}$ ELISA Human BDNF Immunoassay (R\&D Systems Inc.).

This study was conducted in accordance with the Declaration of Helsinki ${ }^{38}$ and ethics approval was received from the Hamilton Integrated Research Ethics Board (study IDs 10-661 and 11-3479); data were collected between March 2011 and November 2014. Altogether, 343 participants were recruited into this study: 250 psychiatric inpatients, and 93 community controls without psychiatric diagnoses (see Study Flow Diagram, Figure 1). This study is reported in accordance with the Strengthening the Reporting of Observational Studies in Epidemiology (STROBE) guidelines. ${ }^{39}$

\section{Statistical Analysis}

Categorical variables were summarized using frequency and percentage. Normally distributed continuous variables were summarized using mean and standard deviation (SD)

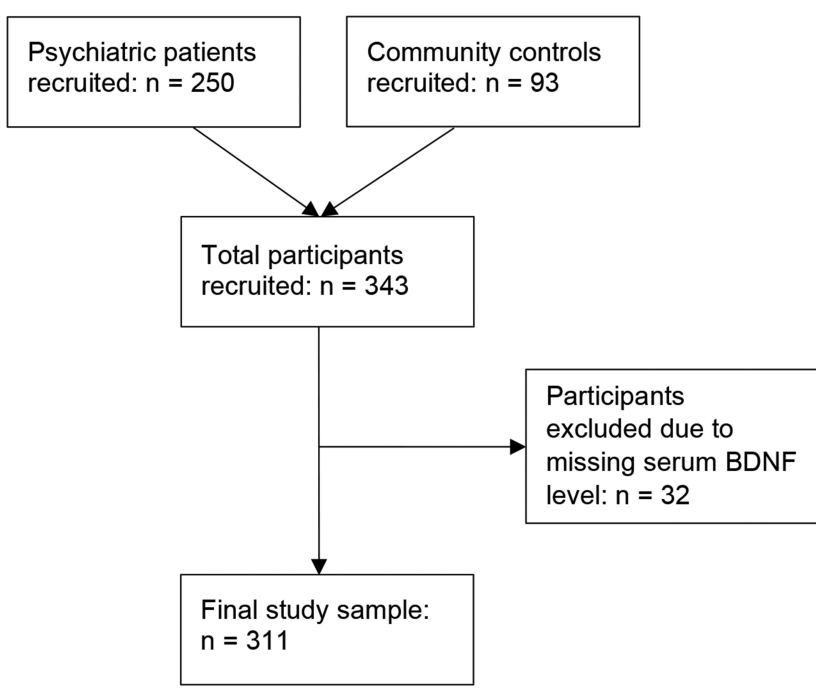

Figure I Study flow diagram.

and non-normally distributed continuous variables were summarized using median and interquartile range (IQR). Serum BDNF values were converted from $\mathrm{pg} / \mathrm{mL}$ to $\mathrm{ng} /$ $\mathrm{mL}$ to achieve greater consistency with previous studies.

Using univariate linear regression analyses, we assessed the associations between serum BDNF levels and modifiable risk factors of interest, including BMI, smoking, antidepressant medication, exercise levels, refined grains intake, fish intake, nut intake, and fruit intake. Food groups were chosen based on the evidence for their impact on BDNF levels in the existing literature. ${ }^{29,30,40,41}$ We then used multivariable regression analyses to adjust for age, sex, and psychiatric group status. Results are reported using estimated betacoefficients with $95 \%$ confidence intervals (CI) and associated $p$ values. Our sample size of 311 participants was adequate to include covariates in the model following the rule of thumb of 10 participants for each covariate included in linear regression analysis. ${ }^{42}$ We identified a single extreme outlier amongst serum BDNF values and conducted a sensitivity analysis excluding this participant.

Our first objective was to explore whether there is an association between serum BDNF levels and modifiable factors such as BMI, smoking, antidepressant medications, exercise levels, and diet within the entire study cohort, including both psychiatric patients and community controls. These factors were chosen due to previous literature finding evidence of their possible association with BDNF levels. ${ }^{29,31-33,43}$

Subsequently, we conducted subgroup analysis to examine whether the findings held within subgroups of psychiatric patients and community controls, and also to investigate any sex-associated differences. All analyses 
were conducted using Stata version 15.1 (StataCorp LP, College Station, TX, USA).

\section{Results}

Altogether, 343 participants were recruited for this study, with a mean age of 45.5 years $(\mathrm{SD}=15.4)$ and $48 \%$ were female. Thirty-two participants were excluded from the analysis due to missing serum BDNF data (see Study Flow Diagram, Figure 1). Participant demographic information can be found in Table 1. Mean serum BDNF levels were $24.05 \mathrm{ng} / \mathrm{mL}(\mathrm{SD}=$ 7.48) for psychiatric inpatients, and $24.77 \mathrm{ng} / \mathrm{mL}(\mathrm{SD}=7.00)$ for community controls. No significant difference in mean serum BDNF levels was found between the groups. Smokers accounted for $39 \%$ of psychiatric inpatients, and $12 \%$ of community controls. A sedentary level of activity was reported by $21 \%$ of psychiatric inpatients, and $10 \%$ of community controls. Mean BMI was 29.3 (SD = 8.8) for psychiatric inpatients, and 27.7 (SD $=6.2$ ) for community controls. Nearly all $(98 \%)$ of psychiatric inpatients were taking psychotropic medications, compared to $22 \%$ of community controls. Further information on the psychotropic medications taken is reported in Table 2.

There was a significant association found between sedentary activity level and lower serum BDNF levels in both univariate (Estimated Beta-coefficient -2.469 , 95\% CI $-4.636,-0.301, p=0.026$; Table 3 ) and fully adjusted models (Estimated Beta-coefficient -2.486 , 95\% CI $-4.698,-0.275, p=0.028$; Table 3). Subgroup analysis further demonstrated that for psychiatric patients this association between sedentary activity and lower serum BDNF level held (Estimated Beta-coefficient -3.057, 95\% CI
-5.418, -0.696, $p=0.011)$. Meanwhile, we did not observe a significant association between sedentary activity and BDNF level for community controls (Estimated Beta-coefficient 1.475, 95\% CI -5.069, 8.020, $p=0.655$ ). We did not find a significant association between BMI, smoking status, antidepressant medication use, and dietary intake, and serum BDNF level in the entire study sample or in either subgroup.

When stratifying results by sex, we found a significant association between sedentary activity level and lower serum BDNF levels for females (Estimated Betacoefficient $-3.178,95 \%$ CI $-6.285,-0.072, p=0.045$; Table 4) but not for males (Estimated Beta-coefficient $-1.415,95 \%$ CI $-4.610,1.779, \mathrm{p}=0.383$; Table 4). We also found that antidepressant medication use was significantly associated with higher serum BDNF levels in males (Estimated Beta-coefficient 3.195, 95\% CI 0.511, 5.880, $\mathrm{p}=0.020$; Table 4) and with lower serum BDNF for females (Estimated Beta-coefficient -3.104, 95\% CI -5.752, $-0.457, \mathrm{p}=0.022$; Table 4).

We conducted a sensitivity analysis removing one outlier case within the serum BDNF values and found that the association between sedentary activity and lower serum BDNF level was similar (Estimated Beta-coefficient $-2.265,95 \%$ CI $-4.383,-0.146, p=0.036)$.

\section{Discussion}

In this study, we found a significant association between sedentary activity, defined as no reported physical activity at work or during leisure, and lower serum BDNF levels in

Table I Participant Demographic and Clinical Characteristics at Baseline $(N=3 \mathrm{II})$

\begin{tabular}{|c|c|c|c|}
\hline Demographics & Total Sample & Psychiatric Patients $n=220$ & Community Controls $n=91$ \\
\hline Age in years; mean (SD) & $45.2(15.6)$ & $44.7(14.6)$ & $46.5(17.9)$ \\
\hline Female sex; n (\%) & $158(50.8)$ & II $3(5 \mathrm{I} .4)$ & $45(49.5)$ \\
\hline Married or common law; n (\%) & III (35.9) & $63(28.9)$ & $48(52.8)$ \\
\hline Unemployment; n (\%) & $191(62.2)$ & I54 (7I.3) & $37(40.7)$ \\
\hline \multicolumn{4}{|l|}{ MINI-diagnosed disorder; n (\%) } \\
\hline Psychotic Disorder ${ }^{\mathrm{a}}$ & $4(1.5)$ & $4(2.2)$ & $0(0.0)$ \\
\hline Mood Disorder ${ }^{b}$ & $90(32.5)$ & $88(46.8)$ & $2(2.3)$ \\
\hline Anxiety Disorder ${ }^{c}$ & $52(19.1)$ & $49(26.6)$ & $3(3.4)$ \\
\hline Eating Disorder ${ }^{d}$ & $\mathrm{I}(0.4)$ & $\mathrm{I}(0.6)$ & $0(0.0)$ \\
\hline Antisocial Personality Disorder ${ }^{\mathrm{e}}$ & $20(7.3)$ & $17(9.2)$ & $3(3.4)$ \\
\hline
\end{tabular}

Notes: a Data available for 272 participants, 183 psychiatric patients and 89 community controls; 'bata available for 277 participants, 188 psychiatric patients and 89 community controls; 'Data available for 273 participants, 184 psychiatric patients and 89 community controls; ${ }^{\mathrm{d} D a t a}$ available for $27 \mathrm{I}$ participants, 182 psychiatric patients and 89 community controls; 'Data available for 274 participants, 185 psychiatric patients and 89 community controls.

Abbreviation: SD, standard deviation. 
Table 2 Participant BDNF Levels and Modifiable Factors at Baseline $(\mathrm{N}=3 \mathrm{I})$

\begin{tabular}{|c|c|c|c|}
\hline Characteristics & Total Sample & Psychiatric Patients $n=220$ & Community Controls $n=91$ \\
\hline Serum BDNF level (ng/mL); mean (SD) & $24.26(7.34)$ & $24.05(7.48)$ & $24.77(7.00)$ \\
\hline $\mathrm{BMI}^{\mathrm{a}}$; mean (SD) & $28.8(8.1)$ & $29.3(8.8)$ & $27.6(6.1)$ \\
\hline Current smoker ${ }^{\mathrm{b}}$; n (\%) & $95(31.4)$ & $84(39.6)$ & $11(12.1)$ \\
\hline \multicolumn{4}{|l|}{ Physical activity ${ }^{\mathrm{c}}$ n (\%) } \\
\hline Sedentary & $53(17.1)$ & $48(21.9)$ & $5(5.5)$ \\
\hline Non-sedentary & $257(82.9)$ & $17 \mid(78.1)$ & $86(94.5)$ \\
\hline Psychotropic Medications; n (\%) & $237(76.2)$ & $217(98.6)$ & $20(22.0)$ \\
\hline Antidepressant & $165(53.1)$ & $|5|(68.6)$ & $14(15.4)$ \\
\hline Antipsychotic & $143(46.0)$ & $142(64.6)$ & $\mathrm{I}(\mathrm{I} . \mathrm{I})$ \\
\hline Mood stabilizer & $99(32.8)$ & $94(42.7)$ & $5(5.5)$ \\
\hline Sedative-hypnotic & $19 \mid(6 \mid .4)$ & $187(85.0)$ & $4(4.4)$ \\
\hline Prazosin & $9(2.9)$ & $8(3.6)$ & $\mathrm{I}(\mathrm{I} . \mathrm{I})$ \\
\hline \multicolumn{4}{|l|}{ Food Intake in servings per day ${ }^{\mathrm{d}}$; mean (SD) } \\
\hline Fruits & $4.73(1.5)$ & $4.63(1.5)$ & $4.96(1.4)$ \\
\hline Refined Grains & $3.5(1.7)$ & $3.44(1.7)$ & $3.65(1.6)$ \\
\hline Fish & $2.64(1.2)$ & $2.55(1.2)$ & $2.84(I . I)$ \\
\hline Nuts & $2.81(1.5)$ & $2.57(0.6)$ & $3.35(1.4)$ \\
\hline
\end{tabular}

Notes: a Data available for 306 participants, 215 psychiatric patients and 91 community controls; ${ }^{b}$ Data available for 303 participants, 212 psychiatric patients and 91 community controls; 'Data available for 310 participants, 219 psychiatric patients and 91 community controls; ${ }^{\mathrm{d} D a t a}$ available for 294 participants, 203 psychiatric patients and 91 community controls.

Abbreviations: SD, standard deviation; BDNF, brain-derived neurotrophic factor; BMI, body mass index.

patients with psychiatric disorders. This finding did not hold within a subgroup of community controls without psychiatric illness. We did not find a significant association between serum BDNF levels and BMI, smoking status, antidepressant medication, or diet. We also found no association between sedentary activity levels and serum BDNF levels in community controls. This finding could suggest that a sedentary lifestyle, which is more common in individuals with diagnosed severe mental illness, ${ }^{44}$ affects individuals with psychiatric illness more than individuals without a psychiatric diagnosis. This could reflect a difference in which BDNF is modulated or expressed in psychiatric patients, or a difference between the effect, quantity, or quality of physical activity between the two groups.

More specifically, we found that sedentary activity was significantly associated with lower serum BDNF levels in females, but not in males. Previous observational studies have provided conflicting evidence for the association between physical activity and serum BDNF levels. ${ }^{26-29}$ Three of these studies found that increased physical activity was associated with decreased serum BDNF levels, ${ }^{26,27,29}$ while another found no significant association. ${ }^{28}$ Of note, the studies that found the significant association had sample populations of mostly or exclusively males, while the last study had a more balanced sample $(\mathrm{N}=75$, female $=$ 47). ${ }^{26-29}$ The results in the present study provide further evidence of a relationship between sex and the association between physical activity and serum BDNF levels that should be explored further in future studies.

It should be noted that a much smaller sample size $(\mathrm{N}=91)$ was involved in the subgroup analysis within community controls. Given that this study involves a secondary analysis of data from the DISCOVER study, a priori sample size calculations were not specifically conducted for our present research question. In conjunction with the 10 variables used in our analyses, this leads to the possibility that there is insufficient power, resulting in an increased likelihood for a type 2 error. Furthermore, we cannot establish any causal relationships given the nature of our observational study design. There was a wide CI for our findings regarding sedentary activity and BDNF levels, which may be due to small sample size or a wide data spread. It is possible that the wide data spread may indicate a variable strength of association between sedentary activity and serum BDNF level between participants. A previous study of 26 participants found that sedentary individuals had a higher serum BDNF levels $(23.63 \pm 2.94$ $\mathrm{ng} / \mathrm{mL}$ ) compared to more active individuals $(19.54 \pm 4.53 \mathrm{ng} /$ 
Table 3 Regression Model of Factors Associated with Serum BDNF Level in All Participants $(\mathrm{N}=3 \mathrm{I} \mathrm{I})$

\begin{tabular}{|c|c|c|c|c|c|c|}
\hline \multirow[t]{2}{*}{ Covariate } & \multicolumn{3}{|l|}{ Unadjusted } & \multicolumn{3}{|c|}{ Adjusted for Age, Sex and Psychiatric Status } \\
\hline & $\begin{array}{l}\text { Estimated Beta- } \\
\text { Coefficient }\end{array}$ & $95 \% \mathrm{Cl}$ & $p$ & $\begin{array}{l}\text { Estimated Beta- } \\
\text { Coefficient }\end{array}$ & $95 \% \mathrm{Cl}$ & $p$ \\
\hline Psychiatric Status & -0.711 & $-2.514,1.091$ & 0.438 & - & - & - \\
\hline Age (years) & -0.033 & $-0.085,0.020$ & 0.218 & - & - & - \\
\hline Female Sex & 1.338 & $-0.297,2.973$ & 0.108 & - & - & - \\
\hline BMI & -0.030 & $-0.132,0.072$ & 0.566 & -0.024 & $-0.127,0.079$ & 0.650 \\
\hline Smoker & 1.352 & $-0.440,3.144$ & 0.139 & 1.638 & $-0.248,3.524$ & 0.088 \\
\hline Sedentary activity & -2.469 & $-4.636,-0.301$ & $0.026^{*}$ & -2.486 & $-4.698,-0.275$ & $0.028 *$ \\
\hline Antidepressant Medications & -0.709 & $-2.35 \mathrm{I},-0.934$ & 0.397 & -0.588 & $-2.479,1.302$ & $0.54 I$ \\
\hline \multicolumn{7}{|l|}{ Food Intake (servings per day) } \\
\hline Fruits & 0.172 & $-0.615,0.960$ & 0.667 & 0.067 & $-0.727,0.860$ & 0.869 \\
\hline Fish & 0.136 & $-0.985,1.257$ & $0.81 \mathrm{I}$ & 0.184 & $-0.946,1.314$ & 0.749 \\
\hline Nuts & 0.509 & $-0.54 \mathrm{I}, \mathrm{I} .560$ & 0.341 & 0.498 & $-0.553,1.549$ & 0.352 \\
\hline Refined Grains & 0.220 & $-0.687,1.127$ & 0.633 & 0.258 & $-0.668,1.185$ & 0.584 \\
\hline
\end{tabular}

Note: *p-value $<0.05$.

Abbreviations: $\mathrm{Cl}$, confidence interval; $\mathrm{BMI}$, body mass index.

$\mathrm{mL})$. Another study of 85 participants demonstrated that individuals performing more than or less than 30 minutes of exercise monthly had serum BDNF levels of $28.75 \mathrm{ng} / \mathrm{mL}$ vs $29.29 \mathrm{ng} / \mathrm{mL}$, respectively, no CIs provided. Nonetheless, to further examine the reasons for our wide CI, we performed a sensitivity analysis by removing one outlier case that fell well outside of previously reported ranges ( 8 to $46 \mathrm{ng} / \mathrm{mL}$ ) for serum BDNF concentrations. ${ }^{4}$ Overall, the p-value of the relationship increased by 0.008 , and the CI decreased by a small margin, which indicated that removing the outlier

Table 4 Age- and Psychiatric Status-Adjusted Regression Model of Factors Associated with Serum BDNF Level Stratified by Sex (N = $3 \mid I)$

\begin{tabular}{|c|c|c|c|c|c|c|}
\hline \multirow[t]{2}{*}{ Covariate } & \multicolumn{3}{|l|}{ Male $(n=153)$} & \multicolumn{3}{|l|}{ Female $(n=158)$} \\
\hline & $\begin{array}{l}\text { Estimated Beta- } \\
\text { Coefficient }\end{array}$ & $95 \% \mathrm{Cl}$ & $p$ & $\begin{array}{l}\text { Estimated Beta- } \\
\text { Coefficient }\end{array}$ & $95 \% \mathrm{Cl}$ & $p$ \\
\hline Psychiatric Status ${ }^{a}$ & -1.897 & $-4.319,0.525$ & 0.124 & 0.000 & $-2.707,2.707$ & 1.000 \\
\hline Age (years) ${ }^{b}$ & -0.072 & $-0.146,0.002$ & 0.055 & -0.001 & $-0.077,0.075$ & 0.982 \\
\hline BMI & -0.164 & $-0.050,0.379$ & 0.132 & 0.072 & $-0.195,0.050$ & 0.244 \\
\hline Smoker & 1.572 & $-0.933,4.077$ & 0.217 & 1.631 & $-1.207,4.468$ & 0.258 \\
\hline Sedentary activity & -1.415 & $-4.609,1.779$ & 0.383 & -3.178 & $-6.285,-0.07 \mathrm{I}$ & $0.045^{*}$ \\
\hline Antidepressant Medications & 3.195 & $0.5 \mathrm{II}, 5.880$ & $0.020 *$ & -3.104 & $-5.752,-0.457$ & $0.022 *$ \\
\hline \multicolumn{7}{|l|}{ Food Intake (servings per day) } \\
\hline Fruits & -0.198 & $-1.279,0.88 \mid$ & 0.717 & 0.324 & $-0.843,1.492$ & 0.584 \\
\hline Fish & -0.162 & $-1.621,1.296$ & 0.826 & 0.550 & $-1.201,2.300$ & 0.536 \\
\hline Nuts & 0.491 & $-0.908,1.890$ & 0.489 & 0.430 & $-1.157,2.018$ & 0.593 \\
\hline Refined Grains & -0.194 & $-1.401,1.013$ & 0.751 & 0.818 & $-0.606,2.243$ & 0.258 \\
\hline
\end{tabular}

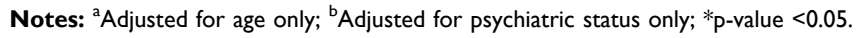

Abbreviations: $\mathrm{Cl}$, confidence interval; $\mathrm{BMI}$, body mass index. 
had minimal impact on the overall relationship between sedentary activity and serum BDNF.

Looking at the other factors investigated in this study, antidepressant use was associated with higher serum BDNF levels in males and lower serum BDNF levels in females. Antidepressant use has been noted to bring BDNF levels back to the baseline of the general population. ${ }^{13}$ However, a meta-analysis by Molendjik et al found that use of specific serotonin reuptake inhibitors (SSRIs) and St. John's wort was associated with high levels of BDNF, while noradrenergic and specific serotonergic antagonists (NaSSAs) were associated with low levels of BDNF (relative to SSRIs and St. John's wort). ${ }^{45}$ This may help explain our results as we did not differentiate between classes of antidepressants. The same meta-analysis noted that studies investigating the link between antidepressants and BDNF levels are generally underpowered, and therefore studies with larger sample sizes must be done before any relationship can be reasonably confirmed. ${ }^{45}$ Future directions for this line of questioning should examine the impact of specific antidepressant medications on BDNF levels and any associated sex differences in this relationship. Regarding food intake, the short Food Frequency Questionnaire that we used is a self-report measure for food intake, and reporting bias might have influenced our results. In addition, the measure grouped food intake of three servings or more per day into the same category. Therefore, it is difficult to discern whether there are differences to be found in serum BDNF levels between individuals consuming a greater number of servings within each food group. Nonetheless, the FFQ is the most commonly used tool to assess the association between diet and disease and is used in numerous studies. ${ }^{37}$ In addition, moderate amounts of certain food groups, such as fruit, are associated with higher serum BDNF levels than higher or lower intake. ${ }^{29}$ While this by itself may indicate a moderation of intake is important in modulating BDNF levels, it could also point to a well-balanced diet as a contributing factor, as individuals who eat a variety of foods will likely eat moderate amounts within each food group. A randomized controlled trial (RCT) found significantly reduced depressive symptoms in their intervention group which was told to eat moderate amounts of fruit, whole grains, nuts, seeds, and fish. ${ }^{46}$ The amounts recommended by Francis et al's study were well below the average intake reported by our participants, which may explain why we did not find significant results. Regarding obesity, while it has previously been linked with lower serum BDNF levels in the context of individuals with type 2 diabetes, our sample size includes many individuals without this diagnosis. ${ }^{47}$ There is also evidence suggesting that anorexia nervosa is associated with decreased serum BDNF levels, ${ }^{48}$ although this did not appear to be the case in the individuals with this diagnosis in our sample.

We did not find a significant association in serum BDNF due to the participants' current smoking statuses. Our sample size of 311 participants is smaller than Jamal et al's study involving 2088 participants which found a positive association between current smoking status and serum BDNF, which may possibly explain the lack of association that we observed. ${ }^{43}$ We also acknowledge that our measure for tobacco use was based on self-report questionnaire, and participants may have chosen to underreport their tobacco use, which is often considered an adverse lifestyle behavior.

To our knowledge, previous studies have found evidence supporting an association between activity levels and serum BDNF levels. However, unlike our study, these studies mainly looked at the effects of acute exercise on serum BDNF as opposed to baseline differences. In addition, common design limitations cited by studies within this topic are limited sample sizes and lack of statistical adjustment for confounding variables. We attempted to address both these limitations with our study. Altogether, 311 participants were in our final study sample, with 220 psychiatric patients and 91 community controls. In comparison, previous observational studies regarding similar research questions had generally much smaller samples (range 26-85). ${ }^{26-29}$ In Szuhany et al's 2015 meta-analysis, the mean sample size of the 29 studies reviewed was just over 38 participants, with the largest sample size being $150 .^{33,49}$ In Molendijk et al's 2014 meta-analysis, only 5 out of 55 studies included in the analysis had sample sizes larger than our sample, although 3 of these studies had far larger sample sizes. ${ }^{32,45,50,51}$ In addition, other studies did not seek to identify differences between psychiatric patients and community controls. ${ }^{33,34}$ We provide comparisons of psychiatric patients and community controls to understand possible differences in the impact of modifiable factors on BDNF levels in these two distinct populations.

Limitations of this study include the cross-sectional design, which precludes the establishment of causal 
relationships from its results. This design was chosen because the DISCOVER study protocol was initially designed to study suicidal behavior, of which incidence is low within the general population. Additionally, given that physical activity was a self-reported measure, the subjective reporting of activity is another limitation. Furthermore, this study involves a secondary analysis of data from the DISCOVER study; therefore, sample size calculations were not conducted specifically for our present research question and the study risks being under-powered to detect differences. Another limitation may include the sample characteristics. $71.3 \%$ and $40.7 \%$ of psychiatric inpatients and community controls were unemployed, respectively; however, many of the community controls were students. These rates are higher than the provincial unemployment rates, which may indicate an unrepresentative sample. ${ }^{52}$ Psychiatric inpatients' employment rates were likely to be affected by their psychiatric status, whereas the majority of the unemployed subjects in the community controls were either students or retired. However, our results showed that age was noted to have an insignificant effect on serum BDNF levels. We note that 32 participants had missing BDNF values and were excluded from the analyses. There were no significant age differences $(p=0.435)$ or sex differences $(p=0.110)$ amongst individuals with and without missing values in general. Amongst participants with missing BDNF values, nearly all missing values occurred in psychiatric patients (94\%) while 6\% occurred in community controls. Notably, psychiatric patients with missing BDNF values were prescribed antidepressants at a lower proportion $(26.7 \%$ versus $68.6 \%$ ) than those who did not have missing values. Although it cannot be definitively confirmed, it appears there may be differences in the clinical profile and treatment of patients with missing values which may bias the findings. Finally, while serum BDNF has been identified as a marker for various aspects of health, there is limited information on whether targeting increased serum BDNF is clinically meaningful in and of itself, or how this would be measured and quantified. This study does not answer questions about whether there is a specific value of serum BDNF one should aim for to achieve optimal health, nor does it answer questions about how quantified changes in serum BDNF affect one's clinical picture. Future studies should examine the relationship between discrete differences in serum BDNF and clinically meaningful outcomes such as symptom improvement, functional improvement, and quality of life.

\section{Conclusion}

In this study, we found a significant association between sedentary activity level and lower serum BDNF levels for patients with psychiatric disorders but not for healthy community controls. In the context of previous literature indicating a link between decreased BDNF and the presence of psychiatric illness, this is a clinically relevant finding and is applicable within mental health treatment settings. With a previously established link between reduced serum BDNF levels and depression, ${ }^{32,53}$ the inclusion of some physical activity into mental wellness or psychiatric treatment plans could be an effective strategy in helping reduce the symptom burden within patients with these conditions. ${ }^{54,55}$ While no definitive causal relationships can be ascertained due to our observational study design, this finding supports the notion that physical activity can provide a positive impact as part of treatment or prevention strategies for psychiatric illness. We found that any level of physical activity is associated with higher serum BDNF levels than sedentary activity. This finding may be helpful for clinicians and treatment programs to support the implementation of supports designed to increase the physical activity of psychiatric patients. Future studies using experimental or longitudinal cohort designs to better examine causal relationships between exercise and serum BDNF levels would be beneficial.

\section{Acknowledgments}

The authors like to thank the study participants for their valuable contributions to research.

\section{Disclosure}

The author reports no conflicts of interest in this work. Funding for DISCOVER study was received in part from the Brain and Behaviour Research Foundation. Dr. Samaan has received research funding from the Canadian Institutes of Health Research.

\section{References}

1. Ohba S, Ikeda T, Ikegaya Y, Nishiyama N, Matsuki N, Yamada MK. BDNF locally potentiates GABAergic presynaptic machineries: target-selective circuit inhibition. Cereb Cortex. 2005;15(3):291-298. doi:10.1093/cercor/bhh130

2. Horch HW, Katz LC. BDNF release from single cells elicits local dendritic growth in nearby neurons. Nat Neurosci. 2002;5 (11):1177-1184. doi:10.1038/nn927

3. Liu PZ, Nusslock R. Exercise-mediated neurogenesis in the hippocampus via BDNF. Front Neurosci. 2018;12(FEB):1-6. doi:10.3389/ fnins.2018.00052 
4. Polacchini A, Metelli G, Francavilla R, et al. A method for reproducible measurements of serum BDNF: comparison of the performance of six commercial assays. Nat Publ Gr. 2015;(March):1-10. doi:10.1038/srep17989

5. Yang B, Ren Q, Zhang JC, Chen QX, Hashimoto K. Altered expression of BDNF, BDNF pro-peptide and their precursor proBDNF in brain and liver tissues from psychiatric disorders: rethinking the brain-liver axis. Transl Psychiatry. 2017;7(5):e1128-e1128. doi: $10.1038 /$ tp. 2017.95

6. Carlino D, De Vanna M, Tongiorgi E. Is altered BDNF biosynthesis a general feature in patients with cognitive dysfunctions? Neuroscientist. $\quad 2013 ; 19(4): 345-353$. doi:10.1177/1073858412 469444

7. Castrén E, Võikar V, Rantamäki T. Role of neurotrophic factors in depression. Curr Opin Pharmacol. 2007;7(1):18-21. doi:10.1016/j coph.2006.08.009

8. Akyol ES, Albayrak Y, Beyazyüz M, Aksoy N, Kuloglu M, Hashimoto K. Decreased serum levels of brain-derived neurotrophic factor in schizophrenic patients with deficit syndrome. Neuropsychiatr Dis Treat. 2015;11:865-872. doi:10.2147/NDT.S79444

9. Khan MS, Wu GWY, Reus VI, et al. Low serum brain-derived neurotrophic factor is associated with suicidal ideation in major depressive disorder. Psychiatry Res. 2019;273 (October2018):108-113. doi:10.1016/j.psychres.2019.01.013

10. Brown DT, Vickers JC, Stuart KE, Cechova K, Ward DD. The BDNF Val66Met polymorphism modulates resilience of neurological functioning to brain ageing and dementia: a narrative review. Brain Sci. 2020;10(4):195. doi:10.3390/brainsci10040195

11. Ng TKS, Ho CSH, Tam WWS, Kua EH, Ho RCM. Decreased serum brain-derived neurotrophic factor (BDNF) levels in patients with Alzheimer's disease (AD): a systematic review and meta-analysis. Int J Mol Sci. 2019;20(2):257. doi:10.3390/ijms20020257

12. Balietti M, Giuli C, Casoli T, Fabbietti P, Conti F. Is blood brain-derived neurotrophic factor a useful biomarker to monitor mild cognitive impairment patients? Rejuvenation Res. 2020;23 (5):411-419. doi:10.1089/rej.2020.2307

13. Sen S, Duman R, Sanacora G. Serum BDNF, depression and anti-depressant medications: meta-analyses and implications. Biol Psychiatry. 2008;64(6):527-532. doi:10.1016/j.biopsych.2008.05.005

14. Naegelin Y, Dingsdale H, Säuberli K, Schädelin S, Kappos L, Barde YA. Measuring and validating the levels of brain-derived neurotrophic factor in human serum. eNeuro. 2018;5(2). doi:10.1523/ENEURO.0419-17.2018

15. Lin CC, Huang TL. Brain-derived neurotrophic factor and mental disorders. Biomed J. 2020;43(2):134-142. doi:10.1016/j. bj.2020.01.001

16. Cattaneo A, Cattane N, Begni V, Pariante CM, Riva MA. The human BDNF gene: peripheral gene expression and protein levels as biomarkers for psychiatric disorders. Transl Psychiatry. 2016;6(11): e958. doi:10.1038/tp.2016.214

17. Eisen RB, Perera S, Banfield L, Anglin R, Minuzzi L, Samaan Z. Association between BDNF levels and suicidal behaviour: a systematic review and meta-analysis. Syst Rev. 2015;4(1). doi:10.1186/s13643-015-0179-z

18. Grah M, Mihanovic M, Ruljancic N, Restek-Petrovic B, Molnar S, Jelavic S. Brain-derived neurotrophic factor as a suicide factor in mental disorders. Acta Neuropsychiatr. 2014;26(6):356-363. doi:10.1017/neu.2014.27

19. Pinheiro RT, Pinheiro KAT, Da Cunha Coelho FM, et al. Brainderived neurotrophic factor levels in women with postpartum affective disorder and suicidality. Neurochem Res. 2012;37 (10):2229-2234. doi:10.1007/s11064-012-0851-9

20. Deveci A, Aydemir O, Taskin O, Taneli F, Esen-Danaci A. Serum BDNF levels in suicide attempters related to psychosocial stressors: a comparative study with depression. Neuropsychobiology. 2007;56 (2-3):93-97. doi:10.1159/000111539
21. Lee J, Nurjono M, Lee TS. Levels of serum brain-derived neurotrophic factor in schizophrenia. J Nerv Ment Dis. 2016;204 (8):636-639. doi:10.1097/NMD.0000000000000463

22. Erickson KI, Miller DL, Roecklein KA. The aging hippocampus: interactions between exercise, depression, and BDNF. Neuroscientist. 2012;18(1):82-97. doi:10.1161/CIRCULA TIONAHA.110.956839

23. Seifert T, Brassard P, Wissenberg M, et al. Endurance training enhances BDNF release from the human brain. Am J Physiol Regul Integr Comp Physiol. 2010;298(2):372-377. doi:10.1152/ajpregu.005 25.2009

24. Heyman E, Gamelin FX, Goekint M, et al. Intense exercise increases circulating endocannabinoid and BDNF levels in humans-Possible implications for reward and depression. Psychoneuroendocrinology. 2012;37(6):844-851. doi:10.1016/j.psyneuen.2011.09.017

25. Jeon YK, Ha CH. The effect of exercise intensity on brain derived neurotrophic factor and memory in adolescents. Environ Health Prev Med. 2017;22(1):1-6. doi:10.1186/s12199-017-0643-6

26. Nofuji Y, Suwa M, Moriyama Y, et al. Decreased serum brain-derived neurotrophic factor in trained men. Neurosci Lett. 2008;437(1):29-32. doi:10.1016/j.neulet.2008.03.057

27. Currie J, Ramsbottom R, Ludlow H, Nevill A, Gilder M. Cardiorespiratory fitness, habitual physical activity and serum brain derived neurotrophic factor (BDNF) in men and women. Neurosci Lett. 2009;451(2):152-155. doi:10.1016/j.neulet.2008.12.043

28. Flöel A, Ruscheweyh R, Krüger K, et al. Physical activity and memory functions: are neurotrophins and cerebral gray matter volume the missing link? Neuroimage. 2010;49(3):2756-2763. doi:10.1016/j.neuroimage.2009.10.043

29. Chan KL, Tong KY, Yip SP. Relationship of serum brain-derived neurotrophic factor (BDNF) and health-related lifestyle in healthy human subjects. Neurosci Lett. 2008;447(2-3):124-128. doi:10.1016/ j.neulet.2008.10.013

30. Araya AV, Orellana X, Espinoza J. Evaluation of the effect of caloric restriction on serum BDNF in overweight and obese subjects: preliminary evidences. Endocrine. 2008;33(3):300-304. doi:10.1007/ s12020-008-9090-X

31. Rosas-Vargas H, Martínez-Ezquerro JD, Bienvenu T. Brain-derived neurotrophic factor, food intake regulation, and obesity. Arch Med Res. 2011;42(6):482-494. doi:10.1016/j.arcmed.2011.09.005

32. Molendijk ML, Spinhoven P, Polak M, Bus BAA, Penninx BWJH, Elzinga BM. Serum BDNF concentrations as peripheral manifestations of depression: evidence from a systematic review and meta-analyses on 179 associations ( $=9484)$. Mol Psychiatry. 2014;19(7):791-800. doi:10.1038/mp.2013.105

33. Szuhany KL, Bugatti M, Otto MW. A meta-analytic review of the effects of exercise on brain-derived neurotrophic factor. $J$ Psychiatr Res. 2015;60:56-64. doi:10.1016/j.jpsychires.2014.10.003

34. Eisen RB, Perera S, Bawor M, et al. Exploring the association between serum BDNF and attempted suicide. Sci Rep. 2016;6 (1):1-8. doi:10.1038/srep25229

35. Samaan Z, Bawor M, Dennis BB, et al. Exploring the Determinants of Suicidal Behavior: conventional and Emergent Risk (DISCOVER): a feasibility study. Pilot Feasibility Stud. 2015;1 (1):1-10. doi:10.1186/s40814-015-0012-4

36. Sheehan DV, Lecrubier Y, Sheehan KH, et al. The mini-international neuropsychiatric Interview (M.I.N.I.): the development and validation of a structured diagnostic psychiatric interview for DSM-IV and ICD-10. J Clin Psychiatry. 1998;59 Suppl 20(Suppl 2):22-57.

37. Iqbal R, Anand S, Ounpuu S, et al. Dietary patterns and the risk of acute myocardial infarction in 52 countries: results of the INTERHEART study. Circulation. 2008;118(19):1929-1937. doi:10.1161/CIRCULATIONAHA.107.738716

38. Declaration of Helsinki World Medical Association Declaration of Helsinki Ethical Principles for medical research involving human subjects. 
39. Vandenbroucke JP, Von Elm E, Altman DG, et al. Strengthening the Reporting of Observational Studies in Epidemiology (STROBE): explanation and elaboration. PLoS Med. 2007;4(10):1628-1654. doi:10.1371/journal.pmed.0040297

40. Molteni R, Barnard RJ, Ying Z, Roberts CK, Gómez-Pinilla F. A high-fat, refined sugar diet reduces hippocampal brain-derived neurotrophic factor, neuronal plasticity, and learning. Neuroscience. 2002;112(4):803-814. doi:10.1016/S0306-4522(02)00123-9

41. Sánchez-Villegas A, Galbete C, Martinez-González MA, et al. The effect of the mediterranean diet on plasma brain-derived neurotrophic factor (BDNF) levels: the PREDIMED-NAVARRA randomized trial. Nutr Neurosci. 2011;14(5):195-201. doi:10.1179/1476830511Y.0 000000011

42. Peduzzi P, Concato J, Kemper E, Holford TR, Feinstein AR. A simulation study of the number of events per variable in logistic regression analysis. J Clin Epidemiol. 1996;49(12):1373-1379. doi:10.1016/s0895-4356(96)00236-3

43. Jamal M, Van der Does W, Elzinga BM, Molendijk ML, Penninx BWJH. Association between smoking, nicotine dependence, and BDNF Val66Met polymorphism with BDNF concentrations in serum. Nicotine Tob Res. 2015;17(3):323-329. doi:10.1093/ntr/ ntu 151

44. Vancampfort D, Firth J, Schuch FB, et al. Sedentary behavior and physical activity levels in people with schizophrenia, bipolar disorder and major depressive disorder: a global systematic review and meta-analysis. World Psychiatry. 2017;16(3):308-315. doi:10.1002/ wps. 20458

45. Molendijk ML, Bus BAA, Spinhoven P, et al. Serum levels of brain-derived neurotrophic factor in major depressive disorder: state-trait issues, clinical features and pharmacological treatment. Mol Psychiatry. 2011;16(11):1088-1095. doi:10.1038/mp.2010.98

46. Francis HM, Stevenson RJ, Chambers JR, Gupta D, Newey B, Lim CK. A brief diet intervention can reduce symptoms of depression in young adults - a randomised controlled trial. PLoS One. 2019;14(10):1-17. doi:10.1371/journal.pone.0222768

47. Krabbe KS, Nielsen AR, Krogh-Madsen R, et al. Brain-derived neurotrophic factor (BDNF) and type 2 diabetes. Diabetologia. 2007;50(2):431-438. doi:10.1007/s00125-006-0537-4
48. Brandys MK, Kas MJH, Van Elburg AA, Campbell IC, Adan RAH. A meta-analysis of circulating BDNF concentrations in anorexia nervosa. World J Biol Psychiatry. 2011;12(6):444-454. doi:10.3109/ 15622975.2011.562244

49. Swift DL, Johannsen NM, Myers VH, et al. The effect of exercise training modality on serum brain derived neurotrophic factor levels in individuals with type 2 diabetes. PLoS One. 2012;7(8):1-7. doi:10.1371/journal.pone.0042785

50. Terracciano A, Lobina M, Piras MG, et al. Neuroticism, depressive symptoms, and serum BDNF. Psychosom Med. 2011;73(8):638-642. doi:10.1097/PSY.0b013e3182306a4f

51. Bus BAA, Tendolkar I, Franke B, et al. Serum brain-derived neurotrophic factor: determinants and relationship with depressive symptoms in a community population of middle-aged and elderly people. World J Biol Psychiatry. 2012;13(1):39-47. doi:10.3109/ 15622975.2010.545187

52. Statistics Canada. Labour force characteristics by province, monthly, unadjusted for seasonality. Labour Force Survey; 2020. Available from: https://www 150.statcan.gc.ca/t1/tbl1/en/tv.action?pid= 1410001702\#tables. Accessed October 9, 2020.

53. Monteleone P, Serritella C, Martiadis V, Maj M. Decreased levels of serum brain-derived neurotrophic factor in both depressed and euthymic patients with unipolar depression and in euthymic patients with bipolar I and II disorders. Bipolar Disord. 2008;10(1):95-100. doi:10.1111/j.1399-5618.2008.00459.x

54. Rethorst CD, Trivedi MH. Evidence-based recommendations for the prescription of exercise for major depressive disorder. J Psychiatr Pract. 2013;19(3):204-212. doi:10.1097/01.pra.000043050 4.16952.3e

55. Kerling A, Kück M, Tegtbur U, et al. Exercise increases serum brain-derived neurotrophic factor in patients with major depressive disorder. J Affect Disord. 2017;215(January):152-155. doi:10.1016/j. jad.2017.03.034
Neuropsychiatric Disease and Treatment

\section{Publish your work in this journal}

Neuropsychiatric Disease and Treatment is an international, peerreviewed journal of clinical therapeutics and pharmacology focusing on concise rapid reporting of clinical or pre-clinical studies on a range of neuropsychiatric and neurological disorders. This journal is indexed on PubMed Central, the 'PsycINFO' database and CAS, and

\section{Dovepress}

is the official journal of The International Neuropsychiatric Association (INA). The manuscript management system is completely online and includes a very quick and fair peer-review system which is all easy to use. Visit http://www.dovepress.com/testimonials.php to read real quotes from published authors.

Submit your manuscript here: https://www.dovepress.com/neuropsychiatric-disease-and-treatment-journal 